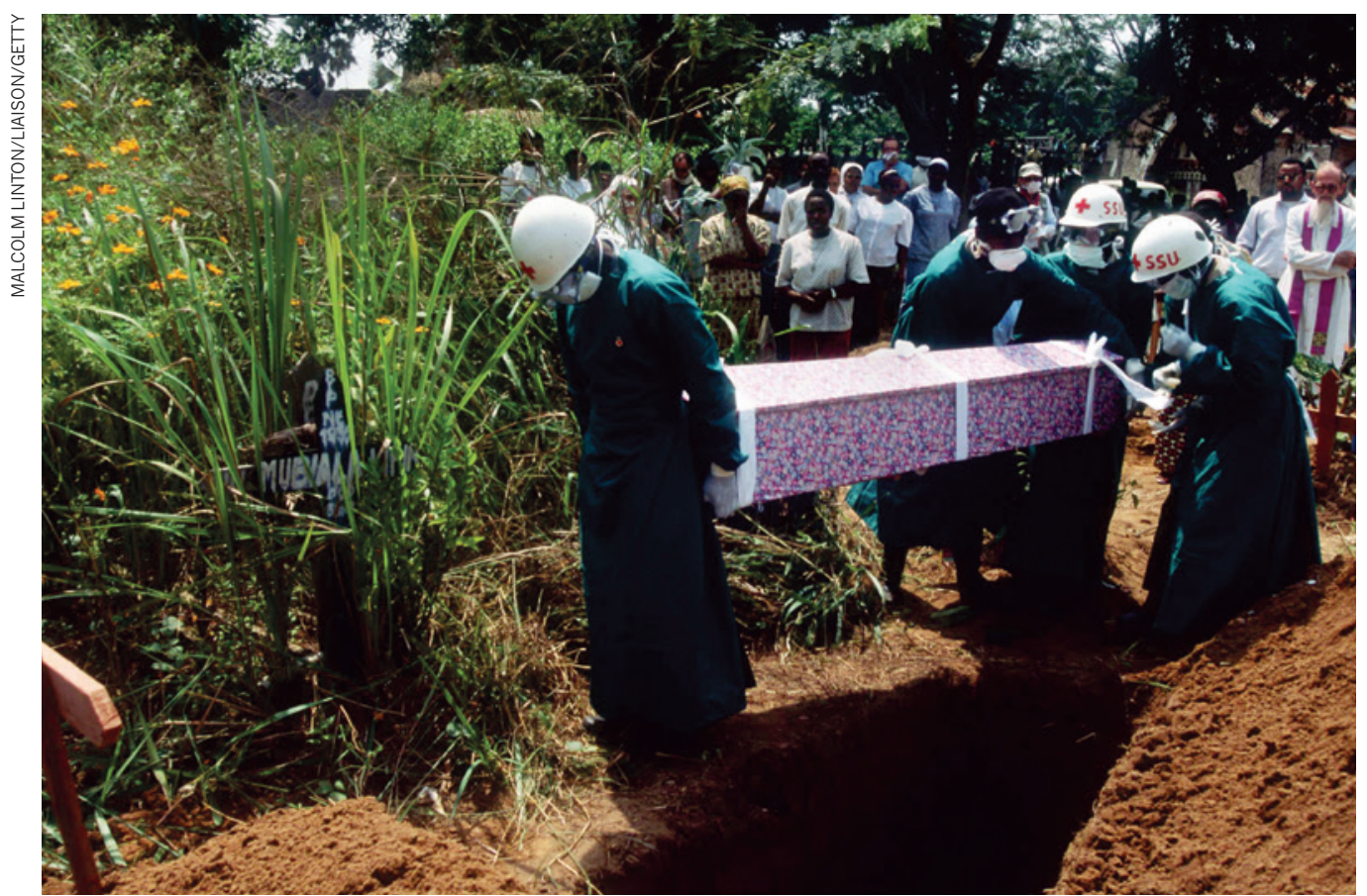

Health workers bury a nun in Kikwit in January 1995, during an Ebola outbreak in Zaire that killed 254 people.

\title{
Ebola: learn from the past
}

\section{Drawing on his experiences in previous outbreaks, David L. Heymann calls for rapid diagnosis, patient isolation, community engagement and clinical trials.}

$\mathrm{T}$ The Ebola outbreak identified in Guinea in March this year has spread to thousands of people across West Africa. Now, in crowded urban areas, transmission has accelerated. The number of confirmed and suspected Ebola cases reported in the week preceding 8 October (854) is more than twice the total number of cases confirmed in the largest previous outbreak, which lasted around four months, beginning in late 2000.

Past outbreaks of Ebola were stopped while they were still in rural areas. Population density was lower, community ties were stronger and, arguably, measures to prevent transmission were easier to implement.

Yet the lessons that I have learned in rural Africa since participating in the investigation of the world's first recorded outbreak of Ebola in 1976 still apply. People with fever and recent contact with an infected person must be diagnosed as soon as possible, and those with Ebola must receive care in isolation wards. Communities need the knowledge and the means to prevent transmission, including safe ways to transport infected people to isolation wards and to handle dead bodies respectfully.

The current outbreak demands all this and more. There is no proven treatment for
Ebola. Amid distrust and battered health-care systems, affected countries and international workers are hoping to launch clinical trials. This outbreak will go on for months yet, and it will not be the last. It would be an injustice not to learn which of several experimental treatments can be used to save lives.

\section{HISTORY LESSONS}

I was a member of the team that investigated the first known outbreak of Ebola ${ }^{1}$. It was in the Democratic Republic of Congo (DRC, then called Zaire) at the Yambuku Mission Hospital nearly four decades ago. By tracing contacts and dates of infection, we searched for the first person infected (possibly from the blood of a game animal butchered for food) who became a source of infection for others. We concluded that this 'index patient' had been treated at the mission clinic for a nosebleed, and for dysentery with an injection. His visit was noted in an unremarkable entry on line 2,355 of an outpatient ledger.

The needles and syringes used at the hospital were shared with the maternity ward. Equipment was, at best, rinsed with distilled water between patients. Outpatients and pregnant women were infected by injections; health workers were infected by blood and bodily fluids from patients, and workers in turn infected family and community members. The virus spread from that one index patient to 318 people, resulting in 280 deaths. The outbreak ended spontaneously, ironically hastened because the hospital closed after workers became infected or fled their posts.

Ebola flared the next year at the Tandala Mission Hospital in the north of the DRC, about 250 kilometres from Yambuku. I was based in Cameroon as an epidemiologist for the US Centers for Disease Control and Prevention. A colleague and I drove for two days across the Cameroon and Central African Republic on unpaved roads through tropical rainforest to investigate.

Although the index case in that instance had also received care at a poorly equipped hospital, a major outbreak did not occur ${ }^{2}$. The physician in charge, a participant in the investigation at Yambuku, had suspected Ebola and isolated the patient, a nine-yearold girl. Only one more infection occurred — in the girl's younger sister — and blood tests of hospital workers and the patient's contacts found that the physician himself was carrying an antibody to Ebola, probably from a previous infection.

These early investigations revealed 
patterns. The first sign is often a cluster of people with diarrhoea or fever, lethargy and other symptoms sometimes confused with typhoid fever. Ebola emerges in rural settings; transmission occurs by contact with infected peoples' blood and body fluids. Its spread is amplified by poor hospital practices (such as re-using needles) - health workers are at great risk of becoming infected and spreading the virus within hospitals and into their communities. Isolation can prevent hospital transmission, assuming that infection controls such as protective equipment and safe disposal practices are in place.

By 1995, when virologist Jean-Jacques Muyembe and I led the response to the Ebola outbreak at Kikwit General Hospital in the $\mathrm{DRC}^{3}$, we knew that rapid and robust action could stop spread, even along major transport links. (Kikwit is 350 kilometres -5 hours by road - from the capital Kinshasa, where one patient travelled to and was rapidly identified and isolated.) We also learned that communities clearly understood the risk of infection, and could be persuaded to forego dangerous funeral rites, such as washing out deceased relatives' mouths or clipping their fingernails.

\section{SUCCESSFUL STRATEGIES}

We had a three-pronged strategy. First, patients were identified and isolated, and protective clothing was provided to health workers. Second, contacts of all patients with Ebola were monitored, and their temperature taken twice a day for three weeks. Those with fever were isolated until diagnosis could be confirmed and those with Ebola were hospitalized. Third, individuals were educated to protect themselves and their families. In this and several other outbreaks, organizations such as the Red Cross and the Red Crescent societies worked with village elders and chiefs to distribute information tailored to local traditions. Red Cross workers in protective gear provided transport for patients and burial services for the dead. When the hospitals were full in Kikwit, some patients were isolated in their homes. Their families were provided with protective clothing and monitored daily.

Other strategies have been less successful. Attempts to block the disease at Africa's porous borders did not stop past outbreaks, and will not work now. A cordon sanitaire was established by the DRC government around Kikwit in 1995 and enforced through military roadblocks. But contacts under fever surveillance travelled outside the cordon in dugout canoes. The military patrolled roads, but not forest paths leading to the Kwilu River.

Fast-forward to 2014. The quarantine of an urban slum of Monrovia, Liberia's capital, was lifted just days after its declaration; it had led to armed clashes, even as residents reportedly moved in and out by avoiding check points or bribing their way through.

Measures that were successful in stopping more than 20 major Ebola outbreaks would have probably worked in March in rural Guinea. These approaches are helping to contain another Ebola outbreak that began in March this year in the DRC. Now, they must be adapted for the more complex, difficult situation in urban, mobile societies. For example, city wards may need to examine all fever cases daily, particularly where contract tracing is ineffective.

\section{TOUGH TRIALS}

The need for standard fluid replacement in patients is now urgent. Rich countries have developed a few experimental vaccines and therapies, spurred in part by fears of bioterrorism. In August, the World Health Organization (WHO) reached a global consensus that it would be ethical for clinical trials of these medicines to be carried out in affected countries, even if the standard regulatory demonstrations of safety and efficacy were not yet complete. Indeed, rigorous clini-

\section{"Survivor serum would not be in short supply."}

cal trials for such treatments are possible only during outbreaks, and are the only way to learn what is effective.

In September, the Wellcome Trust, a biomedical-research charity in London, pledged $£ 3.2$ million (US\$5.2 million) to sponsor clinical trials. This is a fraction of the funding needed, and other funders such as the Bill and Melinda Gates Foundation are joining in. Even with recent undertakings from the international community to send equipment and specialized staff, and to train thousands of health workers, conducting clinical trials will be difficult. The outbreak has closed or overwhelmed health-care facilities, where infected patients have been turned away from hospitals to die at home or in the streets.

Nonetheless, the Wellcome Trust and others are assessing sites for clinical trials, and preparing potential protocols. They are likewise discussing ethical issues such as whether experimental treatments should be made available only in the context of randomized controlled trials, or through other study methodologies that would permit more widespread use.

The number of infected patients far outstrips the limited supplies of candidate vaccines, antivirals and other medicines. Again, experience from history may prove useful. For ten weeks after the Yambuku outbreak in 1976, I stayed on in a 'plasmapheresis' programme. Thirteen Ebola survivors voluntarily supplied their blood plasma, which contained proteins that neutralize the Ebola virus. One of the plasma units was given to a laboratory technician accidentally infected in the United Kingdom; he survived ${ }^{4}$. Almost 20 years later, during the Kikwit outbreak in
1995, eight patients received transfusions of whole blood from survivors; seven lived ${ }^{5}$.

Whether these treatments work cannot be determined from this ad hoc administration of survivor-blood products. Some scientists doubt that they will work, suggesting that immune serum has been shown not to decrease the amount of Ebola virus in the blood. But after reviewing the experimental treatments available, an expert panel convened by the WHO recommended clinical trials using survivors' blood, as did the WHO Blood Regulators Network. Compared with other experimental treatments, plasmapheresis requires a complicated procedure and use of a potentially infectious fluid. But even some rural clinics in sub-Saharan Africa regularly collect blood (the first step in plasmapheresis) for transfusions, and screen out donors who carry HIV or hepatitis.

Unlike the other experimental products, survivor serum would not be in short supply. As results become available, trials could transition into treatment if effectiveness is demonstrated (and health-care facilities reestablished). This could encourage patients and their families to agree to hospitalization, and so help to isolate infected patients and stop the spread of infection. For instance, hundreds of survivors in Sierra Leone, which has so far seen around 2,700 cases of Ebola and about 880 deaths, could supply a plasmapharesis trial enrolling large numbers of patients. In the past, survivors have often been willing to provide blood to help others — that will hopefully be the case again.

\section{AFTER THE HEADLINES}

Research and development for vaccines and treatments must continue once this terrible outbreak has passed. Eventually, as for all emerging infections, attention must shift to prevention at the source - keeping the Ebola virus from breaching the species barrier or using a future vaccine to prevent human infection. Once transmission is fully understood, communities can better prevent it.

For now, full international support must be focused on stopping this outbreak through innovative and intensified patient isolation, contact tracing and community empowerment.

David L. Heymann is professor of infectious disease epidemiology at the London School of Hygiene and Tropical Medicine, UK, and head of the Centre on Global Health Security at Chatham House, London, UK. e-mail:david.heymann@lshtm.ac.uk

1. Breman, J. G. et al. in Ebola Virus Haemorrhagic Fever (ed. Pattyn, S. R.) 86-97 (Elsevier, 1977).

2. Heymann, D. L. et al. J. Infect. Dis. 142, 372-376 (1980).

3. Khan, A. S. et al. J. Infect. Dis. 179, S76-S86 (1999)

4. Emond, R. T. et al. Br. Med. J. 2, 541-544 (1977).

5. Mupapa, K. et al. J. Infect. Dis. 179, (Suppl. 1) S18-S23 (1999). 